(Article)

\title{
Feasibility of Children in Disaster: Evaluation and Recovery (CIDER) Protocol for Traumatized Adolescents in South Korea: A Preliminary Study
}

\author{
Mi-Sun Lee ${ }^{1}$, Hyun Soo Kim ${ }^{2}$, Eun Jin Park ${ }^{3,4}$, and Soo-Young Bhang ${ }^{4,5, *}$ \\ 1 Department of Meditation Psychology, Nungin University, Hwaseong 18582, Korea, \\ dlaltjs1010@hanmail.net \\ 2 Department of Psychiatry, Myongji Hospital, Goyang 10475, Korea, minuchun@hanmail.net \\ 3 Department of Psychiatry, College of Medicine, Inje University, Ilsan Paik Hospital, Goyang 10380, Korea, \\ uma99@daum.net \\ 4 Hallym University Suicide and School Mental Health Institute, Anyang 14066, Korea \\ 5 Department of Psychiatry, Eulji University School of Medicine, Eulji University Hospital, \\ 68 Hangeulbiseok-ro, Nowon-gu, Seoul 01830, Korea, bsy1@eulji.ac.kr \\ * Correspondence: bsy1@eulji.ac.kr; Tel.: +82-2-970-8303; Fax: +82-2-970-8429
}

\begin{abstract}
We aimed to evaluate the feasibility and preliminary efficacy of trauma-focused group psychotherapy in adolescents who experienced traumatic events in Korea. Participants were assigned and recruited from two sites in Korea. Children in Disaster: Evaluation and Recovery (CIDER) V1.0 is a trauma-focused group psychotherapy approach consisting of psychoeducation, normalization, stabilization, and techniques of managing the traumatic memory. The CIDER intervention consists of eight 50-minute-long sessions. The effectiveness of the intervention was evaluated using the Korean version of the Children's Response to Traumatic Events Scale-Revised (K-CRTES-R), the Beck Depression Inventory (BDI), the State Anxiety Inventory for Children (SAIC), and the Pediatric Quality of Life Inventory (PedQL). Data were analyzed by Wilcoxon signed-rank test. We recruited 22 traumatized adolescents (mean age 16 years; SD 1.43; range 1318 years old; $71.4 \%$ boys) in this pilot study. The K-CRTES-R scores were significantly improved $(\mathrm{Z}=-2.85, p<0.01)$. The BDI demonstrated the effectiveness of the therapy $(\mathrm{Z}=-2.35, p<0.05)$. The assessment of the PedQL supported the effect of CIDER $(Z=-3.08, p<0.01)$. However, there was no statistically significant differences in the SAIC scores $(Z=-1.90, p>0.05)$. The results show that there is preliminary evidence that CIDER intervention reduces post-traumatic stress and depressive symptoms and improves quality of life. Our findings indicate that CIDER is feasible for treating adolescents exposed to traumatic events. Larger controlled trials are needed to establish the efficacy of this trauma-focused group psychotherapy and examine its impact on posttraumatic stress disorder.
\end{abstract}

Keywords: CIDER; post-traumatic stress disorder; trauma; adolescent; trauma-focused group psychotherapy

\section{Introduction}

Post-traumatic stress disorder (PTSD) is highly prevalent in children and adolescents who have experienced traumatic events [1]. When these psychological symptoms begin to interfere with daily functioning, school-aged children and adolescents need professional assistance to improve their well-being [2]. For PTSD intervention, trauma-focused cognitive behavioral therapy (TF-CBT) has been recommended by the World Health Organization (WHO) guidelines [3]. Eye movement 
desensitization and reprocessing (EMDR), prolonged exposure (PE), group play therapy, art therapy, school-based intervention, and web-based intervention have been provided for PTSD treatment $[4,5]$. However, insufficient evidence-based therapy is available to recommend using the combination strategy for the intervention of PTSD in children as an alternative treatment [6]. To the best of our knowledge, no reports have been published regarding the Korean version of evidencebased trauma-focused group psychotherapy for adolescents who have experienced traumatic events. Therefore, we evaluated the feasibility and preliminary efficacy of trauma-focused group psychotherapy in Korea.

\section{Methods}

\subsection{Participants and Procedures}

From July to December 2017, participants were enrolled from two sites in an alternative school in South Korea. We recruited adolescents who experienced traumatic events between the ages of 14 and 19 years. The inclusion criteria were that participants (1) be between the ages of 8 and 19 years, (2) had to be currently experiencing PTSD as a result of trauma, and (3) be able to understand and respond to the questionnaire. The exclusion criteria were (1) imminent suicide risk and (2) being under a severe current threat state.

The researchers provided detailed explanations of the research to the participants and their caregivers. Participants and parental consent were required. The parental consent form specified details such as the purpose of the study, the research method, the expected effect, the possibility of withdrawing freely after agreeing to participate, human rights protection, and ethics. After determining potential eligibility through introducing the study, adolescents and parents provided informed consent/assent and participated in a baseline assessment. All procedures were approved by the Eulji University Hospital's Institutional Review Board (IRB No. EMCS 2015-05-021-006).

\subsection{Measures}

\subsubsection{Korean Version of Children's Response to Traumatic Events Scale-Revised (K-CRTES-R)}

The K-CRTES-R consists of 23 items and covers the psychiatric symptom items in the PTSD diagnostic criteria of the Diagnostic and Statistical Manual of Mental Disorders-4th Edition (DSMIV). The subjects should be 6-18 years old, and takes 5-10 min to complete. The K-CRTES-R deals with psychiatric symptoms from the past seven days and uses a four-point Likert scale with 0 representing "not at all", 1 denotes "rarely", 3 denotes "sometimes, and 5 represents "frequent". The overall score ranges from 0 to 115 [7]. In the present study, Cronbach's $\alpha$ was 0.91 .

\subsubsection{Beck Depression Inventory}

The Beck Depression Inventory (BDI), first published in 1961, is a multiple-choice questionnaire self-report inventory. It is one of the most widely used psychometric tests for measuring the severity of depression [8]. It consists of 21 questions about how the subject has been feeling in the last week. Each question has a set of at least four possible responses [9]. When the test is scored, a value of 0 to 3 is assigned for each answer, then the total score is compared to a key to determine depression severity [10]. In this study, Cronbach's $\alpha$ was 0.93 for depression.

\subsubsection{State Anxiety Inventory for Children (SAIC)}

The SAIC is a scale used to measure a child's state anxiety level. It is a version of Speilberger's adult state anxiety inventory [11] that is transformed into a form that children can understand. State anxiety means a temporary state of anxiety caused by the arousal of the autonomic nervous system in a particular situation. It consists of 20 questions. In this study, Cronbach's $\alpha$ was 0.90 for anxiety. 


\subsubsection{Pediatric Quality of Life Inventory (PedQL)}

The PedQL is a tool used to evaluate the reported quality of life in children within a healthcare setting as per the child's self-report or the parent/caregiver's observations [12,13]. In the present study, Cronbach's $\alpha$ was 0.86 .

\subsection{Development of CIDER Protocol V1.0}

Children in Disaster: Evaluation and Recovery (CIDER) V 1.0 was developed in South Korea. It consists of eight 50-minute-long sessions with a child and adolescent group. The goals of the CIDER protocol are as follows: to prevent post-traumatic stress symptoms in high-risk children and adolescents with psychological vulnerability due to a traumatic experience, and to promote the crisis and stress coping skills of children and adolescents who have experienced trauma. After the traumatic event, the sessions help the participants to understand psychoeducation and have an awareness of general psychiatric symptoms. In-depth counseling of children, youths, families, schools, and communities could be linked through intervention. From a long-term perspective, the plan is to rehabilitate children and adolescents and their families. CIDER includes psychosocial interventions that aim to improve post-traumatic stress symptoms that are non-medicinal interventions and can also be targeted toward children and adolescents from high-risk groups exposed to traumatic events. Each component of CIDER is shown in Table 1.

Table 1. The process of Children in Disaster: Evaluation and Recovery (CIDER) V1.0 protocol.

\begin{tabular}{|c|c|c|c|c|}
\hline Session & Title & Key element & Goal & Contents \\
\hline 1 & $\begin{array}{c}\text { From } \\
\text { now on }\end{array}$ & $\begin{array}{l}\text { Psychoeducati } \\
\text { on }\end{array}$ & $\begin{array}{l}\text { Understanding the } \\
\text { definition and } \\
\text { meaning of traumatic } \\
\text { reactions }\end{array}$ & $\begin{array}{l}\text { Guide to the program, sharing the traumatic } \\
\text { responses, education on the definition and } \\
\text { symptoms of trauma, introduction to progress } \\
\text { and intervention, and practice a Subjective } \\
\text { Units of Distress Scale (SUDs) and stabilization } \\
\text { techniques }\end{array}$ \\
\hline 2 & $\begin{array}{l}\text { I'm } \\
\text { alright }\end{array}$ & Stabilization & $\begin{array}{l}\text { Practice how to } \\
\text { control flashback or } \\
\text { dissociation memory }\end{array}$ & $\begin{array}{l}\text { Grounding practice: five areas (visual } \\
\text { grounding, auditory grounding, cognitive } \\
\text { grounding, physical sensory grounding, and } \\
\text { kinesthetic grounding) }\end{array}$ \\
\hline 3 & $\begin{array}{l}\text { As my } \\
\text { heart } \\
\text { goes }\end{array}$ & Containing & $\begin{array}{l}\text { Develop self- } \\
\text { adjustable images } \\
\text { that reduce } \\
\text { discomfort }\end{array}$ & $\begin{array}{l}\text { Describe the concept and purpose of } \\
\text { containing, imagine a containing box, drawing } \\
\text { a containing box, and practice and strengthen } \\
\text { deep breathing }\end{array}$ \\
\hline 4 & $\begin{array}{l}\text { Let's be } \\
\text { free }\end{array}$ & $\begin{array}{l}\text { Emotional } \\
\text { freedom } \\
\text { technique, } \\
\text { safe place }\end{array}$ & $\begin{array}{l}\text { Improvement of } \\
\text { positive affect and } \\
\text { safety and acquiring } \\
\text { self-regulation skills }\end{array}$ & $\begin{array}{l}\text { Emotional freedom techniques (EFTs), setting } \\
\text { a safe place, thinking about sensory elements, } \\
\text { drawing and naming a safe place, practicing } \\
\text { and strengthen deep breathing }\end{array}$ \\
\hline 5 & $\begin{array}{l}\text { My } \\
\text { guardian } \\
\text { angel }\end{array}$ & $\begin{array}{c}\text { Resource } \\
\text { enhancement }\end{array}$ & $\begin{array}{l}\text { Promoting positive } \\
\text { self-confidence and } \\
\text { improving efficiency }\end{array}$ & $\begin{array}{l}\text { Selecting the required resources (personal } \\
\text { resources, supported resources, symbol } \\
\text { resources); finding experiences, figures, and } \\
\text { symbols related to resources; and establishing } \\
\text { and connecting sensory elements of resources }\end{array}$ \\
\hline 6 & $\begin{array}{l}\text { Look } \\
\text { from a } \\
\text { distance }\end{array}$ & Distancing & $\begin{array}{l}\text { Effectively control } \\
\text { intrusive trauma } \\
\text { memory and keep it } \\
\text { realistic }\end{array}$ & $\begin{array}{l}\text { Think about the image or memory you want to } \\
\text { adjust, transform the image, replace it with an } \\
\text { easily handled symbol, and handling memory } \\
\text { with containing methods }\end{array}$ \\
\hline 7 & $\begin{array}{c}\text { Let's } \\
\text { imagine }\end{array}$ & $\begin{array}{l}\text { Imagery } \\
\text { Rehearsal } \\
\text { Therapy }\end{array}$ & $\begin{array}{l}\text { Learn how to control } \\
\text { repeated nightmares } \\
\text { and memories }\end{array}$ & $\begin{array}{l}\text { Thinking of repeat and inconvenient dreams } \\
\text { and memories, drawing dreams or memories, } \\
\text { changing to a positive dream (memory), and } \\
\text { strengthening with breathing }\end{array}$ \\
\hline 8 & Thanks & Future & Understand change & Reminding program process and content, \\
\hline
\end{tabular}




\begin{tabular}{|c|c|c|}
\hline $\begin{array}{l}\text { for your } \\
\text { work }\end{array}$ & template & $\begin{array}{l}\text { and growth and } \\
\text { increase efficiency }\end{array}$ \\
\hline
\end{tabular}

imagine what you want in the future, strengthening with butterfly hugging, and award ceremony

The first session focuses on psychoeducation about understanding the brain and psychological symptoms, the introduction of appropriate treatment, and relaxation skills. The second session consists of stabilization. The third session leads to a better understanding of memory and making a containing box. The fourth session practiced emotional freedom techniques (EFT) and improved a sense of security through safe place exercises. Subsequently, during the fifth session, personal resources, supporting resources, and symbol resources are learned, and sensory elements are strengthened. During the sixth session, participants practice distancing the memory to effectively control the flashback. The seventh session focuses on learning how to change and control repeated and uncomfortable dreams and memories by viewing them as positive experiences. During the closing session, participants examine the process and contents of the program, imagine the desired future situation, and increase their understanding of change and growth. This could subsequently result in improved post-traumatic stress disorder symptoms (Table 1).

\subsection{Data Analyses}

Statistical analyses were conducted using IBM SPSS Statistics Version Ver. 22.0 (SPSS Inc., Chicago, IL, USA). Demographics of participants were analyzed using descriptive statistics. Cronbach's $\alpha$ was computed. Changes in symptoms of PTSD, depression, anxiety, and quality of life from pre- to post-test were evaluated using a Wilcoxon signed-rank test. A $p$-value of $<0.05$ was considered to indicate statistical significance.

\section{Results}

The demographic characteristics of the participants are shown in Table 2. For the evaluation, the demographic data provided included age, sex, school, psychiatric diagnosis, experiences of trauma, academic function, and financial status.

Table 2. Demographics of participants $(n=22)$.

\begin{tabular}{ccc}
\hline Variable & Details & $\boldsymbol{n}$ (\% of Details) \\
\hline Age & Range & $13-18$ \\
& Mean (SD) & $16(1.430)$ \\
\hline Sex & Boy & $16(72.73)$ \\
& Girl & $6(27.27)$ \\
\hline School & Middle & $15(68.18)$ \\
& High & $7(31.82)$ \\
\hline Psychiatric diagnosis * & Attention deficit hyperactivity disorder & $11(50.00)$ \\
& Tourette's syndrome & $2(9.09)$ \\
& Autism spectrum disorder & $3(13.64)$ \\
& Bipolar disorder & $3(13.64)$ \\
& Intellectual disability & $3(13.64)$ \\
& Obsession & $5(22.73)$ \\
& Asperger's syndrome & $1(4.55)$ \\
& Depressive disorder & $11(50.00)$ \\
\hline Experiences of trauma * & Bullying & $13(59.09)$ \\
& School violence & $9(40.91)$ \\
& Domestic violence & $1(4.55)$ \\
& &
\end{tabular}




\begin{tabular}{ccc} 
& Sexual abuse & $2(9.09)$ \\
\hline Academic function & Highest & $1(4.55)$ \\
& High & $1(4.55)$ \\
& Middle & $4(18.18)$ \\
& Low & $7(31.81)$ \\
Status of finance & Lowest & $9(40.91)$ \\
& Highest & $0(0.00)$ \\
& High & $1(4.55)$ \\
& Middle & $14(63.63)$ \\
& Low & $1(4.55)$ \\
& Lowest & $6(27.27)$ \\
\hline
\end{tabular}

${ }^{*}$ Multiple response items; grade response percentages are based on the total sample.

In total, 22 traumatized adolescents (mean age $=16$ years, $\mathrm{SD}=1.43$, range $=13-18$ years) were recruited in this pilot study. Sixteen $(72.73 \%)$ respondents were boys and six $(27.27 \%)$ respondents were girls. More than $68 \%(n=15)$ of the students were in middle school (grade $7-8)$ and $31.82 \%(n=$ 7) were high school students (grade 9-12).

Attention deficit hyperactivity disorder (ADHD) and depressive disorder were the most common psychiatric diagnosis exhibited by the students $(50.00 \%, n=11)$. Around $23 \%$ of students presented obsession $(n=5)$. Diagnoses of autism spectrum disorder (ASD), bipolar disorder, and intellectual disability were reported by $13.64 \%(n=3)$. Tourette's syndrome and Asperger's syndrome were reported by less than $10 \%$.

Most experiences of traumatic events were due to bullying (59.09\%, $n=13$ ). Approximately $41 \%$ of students reported experiences of school violence $(40.91 \%, n=9)$, followed by sexual abuse $(9.09 \%, n=2)$, and domestic violence $(4.55 \%, n=1)$.

More than $40 \%(n=9)$ of students reported academic functioning at the lowest level, and more than $60 \%(n=14)$ reported that they were the middle level of income status (Table 2).

The results showed that the symptoms of PTSD, depression, anxiety, and quality of life, according to the Wilcoxon signed-rank test and the K-CRTES-R, significantly improved $(Z=-2.85, p$ $<0.01)$. The BDI showed the effectiveness of therapy $(Z=-2.35, p<0.05)$, and the assessment of the PedQL supported the effect of CIDER $(Z=-3.08, p<0.01)$. However, there was no statistically significant difference in the SAIC $(Z=-1.90, p>0.05)$ (Table 3).

Table 3. Analysis of the Wilcoxon signed-rank test of variables changes in pre- and post-test $(n=22)$.

\begin{tabular}{|c|c|c|c|}
\hline Variable & & SD) & Z \\
\hline \multirow{2}{*}{ K-CRTES-R } & Pre-test & $20.68(16.78)$ & \multirow{2}{*}{$-2.85^{* *}$} \\
\hline & Post-test & 10.77 (11.96) & \\
\hline \multirow{2}{*}{ BDI } & Pre-test & $13.73(9.62)$ & \multirow{2}{*}{-2.35 * } \\
\hline & Post-test & $9.45(8.38)$ & \\
\hline \multirow{2}{*}{ SAIC } & Pre-test & 35.91 (13.93) & \multirow{2}{*}{-1.90} \\
\hline & Post-test & 32.45 (12.82) & \\
\hline \multirow{2}{*}{ PedQL } & Pre-test & 32.77 (18.52) & \multirow{2}{*}{$-3.08^{* *}$} \\
\hline & Post-test & $20.09(15.90)$ & \\
\hline
\end{tabular}


* BDI: Beck Depression Inventory; K-CRTES-R: Korean Version of Children's Response to Traumatic Events Scale-Revised; SAIC: State Anxiety Inventory for Children; PedQL: Pediatric Quality of Life Inventory.

\section{Discussion}

The ultimate objective of this study was to provide feasible treatment and an evidence-based intervention for PTSD in children and youths. The feasibility and preliminary efficacy of CIDER is supported in psychosocial therapy for adolescents.

These results emphasize that CIDER is effective in reducing the traumatic symptoms experienced by adolescents. The importance of practicing stabilization and normalization skills could decrease PTSD symptoms [14]. Particularly, in the early sessions of CIDER, various stabilization techniques were introduced and practiced, which may have alleviated PTSD symptoms in adolescents who experienced trauma. This was highly associated with previous findings [15] that TF-CBT intervention is associated with statistically significant reductions in posttraumatic stress symptoms $(\mathrm{F}(1.49)=52.708, p<0.001)$ among war-affected, sexually exploited girls compared to the control group. Since there was no control group in the present study, however, it is difficult to gauge whether adolescents' PTSD symptoms might have improved without treatment. Prolonged exposure (PE) was an effective intervention for adolescents $(n=10)$ with diagnostic criteria for PTSD, once again supporting the findings of a previous study [16]. After intensive PE, their PTSD symptom severity and comorbidity were significantly reduced during the three- and six-month follow-ups $(p<0.05)$. Also, neither adverse events nor treatment dropout occurred [16]. This could be useful proof to support clinical trials for the adolescent population. We further need to consider the precautions or adverse events in various clinical settings. Considering these findings, systematic evaluation and treatment against traumatized adolescents must be carefully performed in order to prevent relapse of traumatic symptoms.

From the findings of this study, CIDER intervention is effective in reducing the depression symptoms of adolescents. In the CIDER session, it was possible to mitigate negative emotions and improve positive emotions by exploring and visualizing personal resources, supporting resources, and symbol resources. These results suggest that the intervention is designed to help understand the symptoms of trauma, accept normal reactions, and reduce depressed emotions through image demonstrations [14]. Additionally, the present findings are consistent with another study [17] that showed that acute CBT groups among adolescents $(n=123)$ with major depression or dysthymia reported a greater reduction in self-reported depression and higher recovery rates $(66.7 \%)$ than the waitlist group. Overall, the previous findings [18] that clinical psychotherapy showed significant and similar reductions in the depression symptoms of the 78 adolescent patients with major depressive disorder (MDD) was in line with trends in the present study. Therefore, the findings could support that trauma-focused therapy is an effective intervention for adolescent depression.

CIDER has a positive impact on improving the quality of life of adolescents. As a result of experiencing the CIDER intervention, adolescents who experienced trauma were able to objectively broaden their views on the traumatic events, set plans for the future, and promote psychological well-being. The present study corresponds well with a previous study [19] that children $(n=46,8-$ 12 years old) diagnosed with functional abdominal pain disorders (FAPDs) who received Internet CBT experienced a significant improvement in quality of life compared with the usual treatment group $(n=44)$. We found that Internet CBT could be clinically effective therapy compared with other healthcare services and school systems. This finding appears to support that traumatized adolescents could feel hope for the future when they experience alleviation of post-traumatic stress symptoms [20].

In the present study, treatment had no significant effect on the anxiety symptoms of adolescents exposed to trauma. However, a previous study [21] where 52 children (aged 4-18) were allocated to CBT or EMDR after an explosion accident showed that both treatments substantially reduced children's symptoms of anxiety, depression, post-traumatic stress, and behavior problems. the session durations were similar to our study across therapy conditions, and notably, they 
conducted a joint session with parents when comparing differences with the present study. Therefore, we should consider the need to organize and consider the parts that guide parents and caregivers. The previous study [20] did not find significant differences in the reduction of anxiety symptoms across treatment conditions (TF-CBT group versus therapy usually provided in community clinics) among traumatized youths. Although the results of the present study did not demonstrate statistically significant effects on anxiety symptoms, some of the participants experienced personal academic changes (based on their graduation test) and this should be considered. The general time of the intervention in affecting anxiety in Korean adolescents should be considered, and further studies are crucial to understanding the associations with trauma in adolescents. Preventive mental health services, including school-based educational programs, should be used to treat adolescents who were exposed to traumatic events.

The limitations of the study are related to the limited research and small sample size used. It was difficult to draw conclusions about various trauma types given the small sample size and sampling bias. Thus, this limits the generalizability of findings, as results might differ with the type of exposure to a traumatic event. Also, there was no control group, and a placebo impact could not be ruled out. Therefore, findings are preliminary and must be interpreted with caution.

The results are informative regarding trauma-focused group psychotherapy of school-aged adolescents with PTSD. In conclusion, CIDER is potentially an effective intervention for adolescents with PTSD and provides some benefit for depression, overall stress, and quality of life symptoms, but not anxiety symptoms. For future research, the CIDER protocol could be scaled-up and the system adapted to better suit children and adolescents exposed to traumatic events.

Author Contributions: Conceptualization: S-Y.B. and M-S.L. Data curation: H-S.K. and E.J.P. Formal analysis: M-S.L. and S-Y.B. Investigation: S-Y.B. Methodology: M-S.L., S-Y.B., H-S.K., and E.J.P. Software: S-Y.B. and MS.L. Validation: S-Y.B., H-S.K., and E.J.P. Writing-original draft: M-S.L. Writing-review and editing: S-Y.B. and M-S.L.

Funding: This study was supported by a grant from the Korean Mental Health Technology R\&D Project, Ministry of Health and Welfare, Republic of Korea (HM15C1058).

Conflicts of Interest: The authors have no potential conflicts of interest to disclose.

\section{References}

1. Gillies, D.; Taylor, F.; Gray, C.; O’Brien, L.; D'Abrew, N. Psychological therapies for the treatment of posttraumatic stress disorder in children and adolescents (Review). Evid. Based Child Health 2013, 8, 1004-1116.

2. Munthe-Kaas, H.M.; Johansen, S.; Blaasvaer, N.; Hammerstrom, K.T.; Nilsen, W. The Effect of Psychosocial Interventions for Preventing and Treating Depression and Anxiety among at-Risk Children and Adolescents; Norwegian Institute of Public Health: Oslo, Norway, 2014.

3. World Health Organization. Guidelines for the Management of Conditions Specifically Related to Stress; WHO: Geneva, Switzerland, 2013.

4. $\quad$ Lee, M.S.; Hwang, J.W.; Lee, C.S.; Kim, J.Y.; Lee, J.H.; Kim, E.; Chang, H.Y.; Bae, S.; Park, J.H.; Bhang, S.Y. Development of post-disaster psychosocial evaluation and intervention for children: Results of a south korean delphi panel survey. PLOS ONE 2018, 13, e0195235.

5. Gillies, D.; Maiocchi, L.; Bhandari, A.P.; Taylor, F.; Gray, C.; O’Brien, L. Psychological therapies for children and adolescents exposed to trauma. Cochrane Database Syst. Rev. 2016, 10, CD012371.

6. (NICE) NIfCE. Post-Traumatic Stress Disorder: The Management of PTSD in Adults and Children in Primary and Secondary Care. National Clinical Practice Guideline 2005. Available online: http://www.nice.org.uk/nicemedia/live/10966/29769/29769.pdf (accessed on May 27 2018).

7. Napper, L.E.; Fisher, D.G.; Jaffe, A.; Jones, R.T.; Lamphear, V.S.; Joseph, L.; Grimaldi, E.M. Psychometric properties of the child's reaction to traumatic events scale-revised in english and lugandan. J. Child Fam. Stud. 2015, 34, 1285-1294.

8. Beck, A.T.; Ward, C.H.; Mendelson, M.; Mock, J.; Erbaugh, J. An inventory for measuring depression. Arch. Gen. Psychiatry 1961, 4, 561-571.

9. Richter, P.; Werner, J.; Bastine, R.; Heerlein, A.; Kick, H.; Sauer, H. Measuring treatment outcome by the 
beck depression inventory. Psychopathology 1997, 30, 234-240.

10. Saal, W.; Kagee, A.; Bantjes, J. Utility of the beck depression inventory in measuring major depression among individuals seeking hiv testing in the western cape, south africa. AIDS Care 2018, 30, 29-36.

11. Spielberger, C.D. Manual for State-Trait Anxiety Inventory for Children; Consulting Psychologist Press: Palo Alto, CA, USA, 1972.

12. Desai, A.D.; Zhou, C.; Stanford, S.; Haaland, W.; Varni, J.W.; Mangione-Smith, R.M. Validity and responsiveness of the pediatric quality of life inventory (pedsql) 4.0 generic core scales in the pediatric inpatient setting. JAMA Pediatr. 2014, 168, 1114-1121.

13. Buck, D.; Clarke, M.P.; Powell, C.; Tiffin, P.; Drewett, R.F. Use of the pedsql in childhood intermittent exotropia: Estimates of feasibility, internal consistency reliability and parent-child agreement. Qual. Life Res. 2012, 21, 727-736.

14. Birkhead, G.S.; Vermeulen, K. Sustainability of psychological first aid training for the disaster response workforce. Am. J. Public Health 2018, 108, S381-S382.

15. O'Callaghan, P.; McMullen, J.; Shannon, C.; Rafferty, H.; Black, A. A randomized controlled trial of trauma-focused cognitive behavioral therapy for sexually exploited, war-affected congolese girls. J. Am. Acad. Child Adolesc. Psychiatry 2013, 52, 359-369.

16. Hendriks, L.; de Kleine, R.A.; Heyvaert, M.; Becker, E.S.; Hendriks, G.J.; van Minnen, A. Intensive prolonged exposure treatment for adolescent complex posttraumatic stress disorder: A single-trial design. J. Child Psychol. Psychiatry 2017, 58, 1229-1238.

17. Clarke, G.N.; Rohde, P.; Lewinsohn, P.M.; Hops, H.; Seeley, J.R. Cognitive-behavioral treatment of adolescent depression: Efficacy of acute group treatment and booster sessions. J. Am. Acad. Child Adolesc. Psychiatry 1999, 38, 272-279.

18. Brent, D.A.; Holder, D.; Kolko, D.; Birmaher, B.; Baugher, M.; Roth, C.; Iyengar, S.; Johnson, B.A. A clinical psychotherapy trial for adolescent depression comparing cognitive, family, and supportive therapy. Arch. Gen. Psychiatry 1997, 54, 877-885.

19. Lalouni, M.; Ljotsson, B.; Bonnert, M.; Ssegonja, R.; Benninga, M.; Bjureberg, J.; Hogstrom, J.; Sahlin, H.; Simren, M.; Feldman, I.; et al. Clinical and cost effectiveness of online cognitive behavioral therapy in children with functional abdominal pain disorders. Clin. Gastroenterol. Hepatol. 2018, doi:10.1016/j.cgh.2018.11.043.

20. Jensen, T.K.; Holt, T.; Ormhaug, S.M.; Egeland, K.; Granly, L.; Hoaas, L.C.; Hukkelberg, S.S.; Indregard, T.; Stormyren, S.D.; Wentzel-Larsen, T. A randomized effectiveness study comparing trauma-focused cognitive behavioral therapy with therapy as usual for youth. J. Clin. Child Adolesc. Psychol. 2014, 43, 356369.

21. de Roos, C.; Greenwald, R.; den Hollander-Gijsman, M.; Noorthoorn, E.; van Buuren, S.; de Jongh, A. A randomised comparison of cognitive behavioural therapy (cbt) and eye movement desensitisation and reprocessing (emdr) in disaster-exposed children. Eur. J. Psychotraumatol. 2011, 2, doi:10.3402/ejpt.v2i0.5694. 